J. Lake Sci. (湖泊科学), 2015, 27(1): 50-57

http: //www. jlakes.org. E-mail : jlakes@niglas.ac.cn

(C) 2015 by Journal of Lake Sciences

\title{
有机质对城市污染河道沉积物铵态氮吸附一解吸的影响"
}

\author{
刘 波 ${ }^{1,2}$, 盛 明 $^{1}$, 唐 千 $^{1}$, 刘 娜 $^{1}$, 吴馨婷 ${ }^{1}$, 朱程芳 ${ }^{1}$, 沈玲玲 ${ }^{1}$, 周 准 $^{1}$, 王国祥 ${ }^{2 * *}$ \\ ( 1 : 南通大学地理科学学院, 南通 226007) \\ $(2$ : 江苏省环境演变与生态修复重点实验室, 南京 210046)
}

摘 要: 采集污染程度不同的城市河道沉积物 (通吕运河、濠河和通甲河), 在分析 $\mathrm{H}_{2} \mathrm{O}_{2}$ 对沉积物有机质和铵态氮影响的 基础上, 分析沉积物在去除有机质前后铵态氮释放动力学和吸附热力学过程, 研究城市污染河道沉积物有机质对铵态氮 吸附一解吸的影响. 结果表明: 单位体积 $\mathrm{H}_{2} \mathrm{O}_{2}$ 对有机质去除率随 $\mathrm{H}_{2} \mathrm{O}_{2}$ 使用量增多而降低; 去除有机质后, 沉积物铵态氮含 量显著增加, 通吕运河、濠河和通甲河铵态氮最大含量分别是有机质去除前的 4.16、3. 55 和 2.85 倍; 沉积物对铵态氮的 饱和吸附量随有机质含量减少而下降; 沉积物铵态氮释放过程均表现为先快速释放, 后减缓至平衡过程; 去除有机质后, 随着有机质含量的减少, 沉积物铵态氮的最大释放量呈增大趋势; 沉积物有机质和铵态氮含量是影响沉积物铵态氮释放 的主要因素.

关键词: 城市河道; 有机质; 沉积物; 铵态氮; 吸附 - 解吸

\section{Effect of organic matter on adsorption/desorption of ammonium in the sediments of heav- ily polluted urban river}

LIU Bo ${ }^{1,2}$, SHENG Ming ${ }^{1}$, TANG Qian ${ }^{1}$, LIU Na ${ }^{1}$, WU Xinting ${ }^{1}$, ZHU Chengfang ${ }^{1}$, SHEN Lingling ${ }^{1}$, ZHOU Zhun $^{1} \&$ WANG Guoxiang ${ }^{2}$

(1: School of Geographical Science, Nantong University, Nantong 226007, P. R. China)

(2: Jiangsu Key Laboratory of Environmental Change \& Ecological Construction, Nanjing 210046, P. R. China)

Abstract : The effect of organic matter on ammonium release kinetics and adsorption isotherms of the sediments from different trophic level urban rivers (named TL, HH and TJ) and its mechanism was discussed. The results showed the removal ratio of organic matter of per unit volume of $\mathrm{H}_{2} \mathrm{O}_{2}$ tended to decrease with volume of $\mathrm{H}_{2} \mathrm{O}_{2}$ increasing. The content of ammonium in sediments tended to increase significantly as sediment organic matter was removed by $\mathrm{H}_{2} \mathrm{O}_{2}$. Ammonium adsorption capacity tended to decrease with their organic matter contents decreasing. The equilibrium time of ammonium release was prolonged with the increasing of the organic matter contents in the studied sediment, and the amounts of the ammonium released increased first and then reached equilibrium gradually. Removal of organic matter could increase ammonium release capacity. The main factors affecting ammonium release were analyzed. Organic matter content and ammonium concentration were the key factors on ammonia release from sediments. Keywords: Urban river; organic matter; sediment; ammonium; adsorption/desorption

氮素含量过高是我国城市污染河道水体环境的主要特征. 沉积物作为排人河道中各类污染物的主要归 宿, 累积了大量的含氮污染物质. 富集于沉积物的有机质经矿化后产生的铵态氮绝大部分会被沉积物颗粒 所吸附 ${ }^{[1]}$, 在一定条件下, 又会通过解吸作用重新释放到水相中, 成为二次污染源 ${ }^{[2-3]}$. 研究表明, 内源氮已 成为水体氮素污染的主要贡献者 ${ }^{[4]}$. 因此, 沉积物对铵态氮的吸附 - 解吸过程是影响污染河道氮负荷的一个 重要过程, 对科学评估河道氮负荷及其生态效应具有重要作用 ${ }^{[5]}$.

* 国家自然科学基金项目 (41173078)、江苏省自然科学基金项目 (SBK201321353)、南通市自然科学基金项目 (BK2013058) 和大学生创新训练计划项目 (201310304038Z,201310304069Y) 联合资助. 2014-01 -04 收稿;201404-23 收修改稿. 刘波(1976 ), 男,博士, 副教授; E-mail:lb@ ntu. edu. cn.

** 通信作者;E-mail: wangguoxiang@ njnu. edu. cn. 
沉积物对铵态氮的吸附一解吸与沉积物有机质含量密切相关, 但是至今并没有取得明确的结论. 侯立 军、Boatman 、刘敏等 ${ }^{[5-7]}$ 认为有机质含量与铵态氮吸附呈正相关,在有机质含量高的沉积物中, 有机质制约着 沉积物铵态氮的吸附一解吸行为. Holmboe、Alongi 等 ${ }^{[8-9]}$ 则得到了相反的结果, 认为有机质对矿物质的包裹性 随有机质含量增多而增强, 进而会阻塞更多的铵态氮吸附点位, 导致沉积物对铵态氮的吸附与固定能力减 弱. 由于自然环境中影响有机质矿化和铵态氮吸附一解吸的因素众多,因而很难在自然条件下获得矿物结构 相同、有机质含量不同的沉积物, 或许这是造成上述分歧的主要原因. Nam 等 ${ }^{[10]}$ 发现 $\mathrm{H}_{2} \mathrm{O}_{2}$ 可以去除土壤中 部分有机质, 且对土壤类型和矿物成分影响较小. 梁重山等 ${ }^{[11]}$ 证明经 $\mathrm{H}_{2} \mathrm{O}_{2}$ 处理的土壤样品, 可以克服土壤 物理结构的变化对菲吸附一解吸过程的影响. 鉴于此,国内许多学者采用这一方法,探讨了土壤有机质含量 对重金属 ${ }^{[12-14]}$ 、土需素 ${ }^{[15]}$ 等物质吸附一解吸的影响. 王圣瑞等 ${ }^{[16]}$ 用 $\mathrm{H}_{2} \mathrm{O}_{2}$ 法探讨了富营养化湖泊沉积物有机 质对铵态氮吸附一解吸的影响, 发现随着有机质含量被去除, 有机质的极性集团逐渐减少, 导致对铵态氮吸 附能力减弱. 该研究还发现, 随着有机质被 $\mathrm{H}_{2} \mathrm{O}_{2}$ 去除, 可转化态氮显著增加. 但是, 关于增加的可转化态氮对 铵态氮吸附一解吸的影响并没有做深人探讨. 本研究选取典型城市污染河道沉积物, 对比分析经 $\mathrm{H}_{2} \mathrm{O}_{2}$ 预处理 和未经预处理的不同有机质含量沉积物的铵态氮释放动力学与吸附热力学过程, 探讨城市污染河道沉积物 有机质对铵态氮吸附一解吸的影响机制.

\section{1 材料与方法}

\section{1 沉积物与水样采集}

选取南通市区内污染程度不同的河道, 分别是通吕运河 ( TL)、濠河 ( HH) 和通甲河 ( TJ), 见图 1. 通吕运 河是南通第一运河, 具有航运、灌溉、行洪等功能,污染程度较低; 濠河是南通著名的风景名胜区, 有少量的生 活污水汇人; 通甲河为生活污水受纳水体, 污染严重. 选取的 3 条河流所处的地理位置相近, 影响的自然因素 差异不大. 用自制柱状沉积物采样器于 7 月 4 日分别在选定河道处采集沉积物, 同步采集上覆水,迅速运回 实验室,进行上覆水溶解氧 $(\mathrm{DO}) 、 \mathrm{pH}$ 、高锰酸盐指数 $\left(\mathrm{COD}_{\mathrm{Mn}}\right)$ 、总氮 $(\mathrm{TN})$ 、铵态氮 $\left(\mathrm{NH}_{4}^{+}-\mathrm{N}\right)$ 、硝态氮 $\left(\mathrm{NO}_{3}^{-}-\mathrm{N}\right)$ 、亚硝态氮 $\left(\mathrm{NO}_{2}^{-}-\mathrm{N}\right)$ 以及间隙水和沉积物的有机质含量 $(\mathrm{OM}) 、 \mathrm{TN} 、 \mathrm{NH}_{4}^{+}-\mathrm{N}^{-} \mathrm{NO}_{3}^{-}-\mathrm{N}^{-} \mathrm{NO}_{2}^{-}-\mathrm{N}$ 的测 定. 采集的表层沉积物经过低温干燥过 100 目耖,备用.

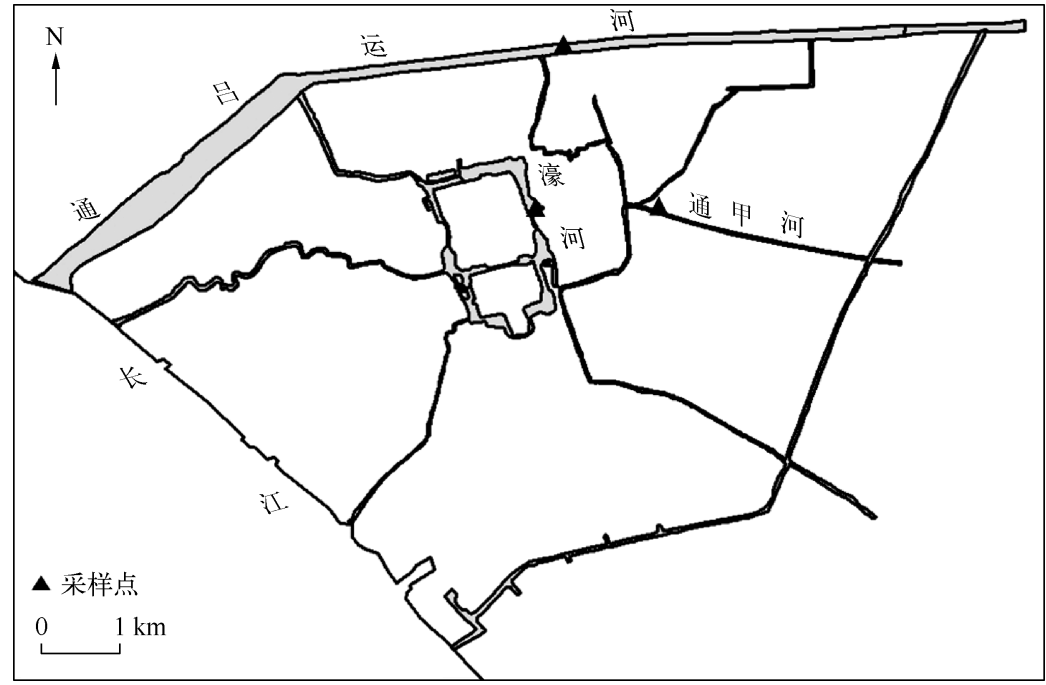

图 1 南通市采样点示意

Fig. 1 Distribution of sampling sites in Nantong City

\section{2 方法}

1.2.1 样品制备 每次分别取 $2 \mathrm{~g}$ 各采样点备用沉积物样品于若干烧杯中 (水土比为 $10: 1$ ). 在 6 个烧杯中 
分别加人 $0 、 1 、 2 、 4 、 5 、 7 \mathrm{ml}$ 的 $30 \% \mathrm{H}_{2} \mathrm{O}_{2}$, 摇匀. 在 $35^{\circ} \mathrm{C}$ 恒温水浴中反应, 待没有气泡生成后, 将烧杯置于电炉 加热至沸腾, 去除多余 $\mathrm{H}_{2} \mathrm{O}_{2}$ 后, 将混合液冷冻干燥. 待样品干燥后过 100 目笁, 备用. 测定有机质和铵态氮含 量. 以上实验在相同条件下作 3 次平行, 相对误差低于 $5 \%$.

1.2 .2 铵态氮释放动力实验 分别称取 $1 \mathrm{~g}$ 经 $\mathrm{H}_{2} \mathrm{O}_{2}$ 处理和备用自然沉积物样品置于 $100 \mathrm{ml}$ 离心管中, 加人 $0.02 \mathrm{~mol} / \mathrm{L}$ 的 $\mathrm{KCl}$ 溶液 $50 \mathrm{ml}$, 在室温下 $\left(22 \pm 1{ }^{\circ} \mathrm{C}\right)$ 以 275 转 $/ \mathrm{min}$ 振荡, 每隔一定时间间隔 $(5 、 15 、 30 、 60$ 、 $120 、 240 、 360 \mathrm{~min}$ ) 取出, 在 5000 转 $/ \mathrm{min}$ 条件下离心 $10 \mathrm{~min}$, 取出 $10 \mathrm{ml}$ 上清液, 测定上清液铵态氮浓度. 以 上实验在相同条件下作 3 次平行,相对误差低于 $5 \%$.

1.2 .3 铵态氮吸附热力学实验 称取原沉积物制备样品 ( TL、 $\mathrm{HH} 、 \mathrm{TJ}$ ) 和经 $7 \mathrm{ml} \mathrm{H}_{2} \mathrm{O}_{2}$ 处理的样品 ( $\mathrm{TLH} 、 \mathrm{HHH} 、$ $\mathrm{TJH}$ ) 各 $2.5 \mathrm{~g}$ 置于 $100 \mathrm{ml}$ 聚乙烯塑料离心管中, 分别加人 $25 \mathrm{ml}$ 不同浓度的铵态氮标准溶液 (初始质量浓度 序列为 $50 、 100 、 150 、 200 、 250 、 300 、 350 、 400 、 450 、 500 \mathrm{mg} / \mathrm{L})$, 在恒温振荡器上振荡吸附 $12 \mathrm{~h}\left(22 \pm 1{ }^{\circ} \mathrm{C}\right)$, 静置 平衡 $2 \mathrm{~h}$, 取上清液过 $0.45 \mu \mathrm{m}$ 孔径的微孔滤膜抽滤. 以上实验在相同条件下作 3 次平行, 相对误差低 于 $5 \%$.

\section{3 样品分析}

用 $2 \mathrm{~mol} / \mathrm{L}$ 的 $\mathrm{KCl}$ 溶液浸提 (液:土 $=5: 1$ ), 振荡 $30 \mathrm{~min}$, 过滤后用纳氏比色法 (波长为 $420 \mathrm{~nm}$ ) 测定浸 提液中 $\mathrm{NH}_{4}^{+}-\mathrm{N}$ 浓度 ${ }^{[17]}$. 有机质含量采用重铬酸钾 - 硫酸外加热法测定. 铵态氮、高锰酸盐指数采用标准方法 测定.

\section{4 计算方法}

为了定量分析铵态氮释放动力学过程, 用一级动力学方程描述不同实验条件下表层沉积物铵态氮的释 放动力学过程 ${ }^{[5,18]}$,公式为:

$$
N_{t}=N_{0}\left(1-\mathrm{e}^{-k t}\right)
$$

式中, $N_{t}$ 为 $t$ 时刻的铵态氮释放量 $(\mathrm{mg} / \mathrm{kg}), N_{0}$ 为释放平衡时铵态氮的释放量 (最大释放量) $(\mathrm{mg} / \mathrm{kg}), k$ 为铵 态氮释放速率常数, $t$ 为释放时间 $(\min )$.

采用 Langmuir 吸附方程来定量描述沉积物不同有机质组成对铵态氮的吸附特征, 公式为:

$$
Q_{\mathrm{e}}=Q_{\mathrm{m}} b C_{\mathrm{e}} /\left(1+b C_{\mathrm{e}}\right)
$$

式中, $Q_{\mathrm{m}}$ 为单位质量沉积物对铵态氮的饱和吸附量 $(\mathrm{mg} / \mathrm{kg}), b$ 为吸附系数, $Q_{\mathrm{e}}$ 为单位质量沉积物对铵态氮 的吸附量 $(\mathrm{mg} / \mathrm{kg}), C_{\mathrm{e}}$ 为平衡溶液铵态氮质量浓度 $(\mathrm{mg} / \mathrm{L})$.

\section{2 结果与分析}

\section{2. $1 \mathrm{H}_{2} \mathrm{O}_{2}$ 对沉积物有机质及铵态氮的作用}

随着 $\mathrm{H}_{2} \mathrm{O}_{2}$ 使用量的增加, 经不同量 $\mathrm{H}_{2} \mathrm{O}_{2}$ 处理的各组沉积物有机质含量均表现出下降的趋势. 当 $\mathrm{H}_{2} \mathrm{O}_{2}$ 使用量为 $7 \mathrm{ml}$ 时, 有机质含量最低, 有机质去除率分别为 $40.82 \% 、 83.60 \%$ 和 $65.05 \%$. 从有机质含量变化 过程来看, 单位体积 $\mathrm{H}_{2} \mathrm{O}_{2}$ 对有机质去除率随 $\mathrm{H}_{2} \mathrm{O}_{2}$ 使用量增多而降低, TL、 $\mathrm{HH}$ 和 TJ 实验组在 $\mathrm{H}_{2} \mathrm{O}_{2}$ 使用量为 $2 \mathrm{ml}$ 时, 每毫升 $\mathrm{H}_{2} \mathrm{O}_{2}$ 对有机质去除率分别为 $17.73 \% 、 33.36 \%$ 和 $27.23 \%$, 此时有机质去除率分别已达到各 组最高去除率的 $86.84 \%$ 、 $79.80 \%$ 和 $83.72 \%$.

各实验组沉积物铵态氮随着 $\mathrm{H}_{2} \mathrm{O}_{2}$ 使用量的增加表现出增加的趋势 (图 2), 与有机质变化过程相反, 这 与王圣瑞等的研究结果相似 ${ }^{[16]}$. 有机质含量较高的 $\mathrm{TJ}$ 组和 $\mathrm{HH}$ 组铵态氮含量随 $\mathrm{H}_{2} \mathrm{O}_{2}$ 使用量增多而增加的 过程更为明显, 尤其是 $\mathrm{TJ}$ 组表现出近似线性的增加过程. 当 $\mathrm{H}_{2} \mathrm{O}_{2}$ 使用量为 $7 \mathrm{ml}$ 时, $\mathrm{HH}$ 组和 $\mathrm{TJ}$ 组铵态氮达 到最大值, 分别比处理前增加了 3.55 和 2.85 倍. 有机质含量较低的 TL 组, 在 $\mathrm{H}_{2} \mathrm{O}_{2}$ 使用量为 $4 \mathrm{ml}$ 时, 铵态 氮含量最高, 为 $44.13 \pm 3.48 \mathrm{mg} / \mathrm{kg}$, 为处理前的 4.16 倍. 将有机质含量与铵态氮含量作了相关性分析, 结果 发现, 沉积物本底有机质含量较高的 TJ 组和 HH 组, 两者之间存在显著的负相关关系 $(P<0.05)$, 有机质含 量较低的 TL 组两者相关性不显著 $(P>0.05)$ (图 2).

\section{2 有机质对沉积物铵态氮释放动力学的影响}

2.2.1 经 $\mathrm{H}_{2} \mathrm{O}_{2}$ 处理沉积物 经 $\mathrm{H}_{2} \mathrm{O}_{2}$ 处理的不同有机质含量的沉积物铵态氮释放过程表现出先快速释放, 

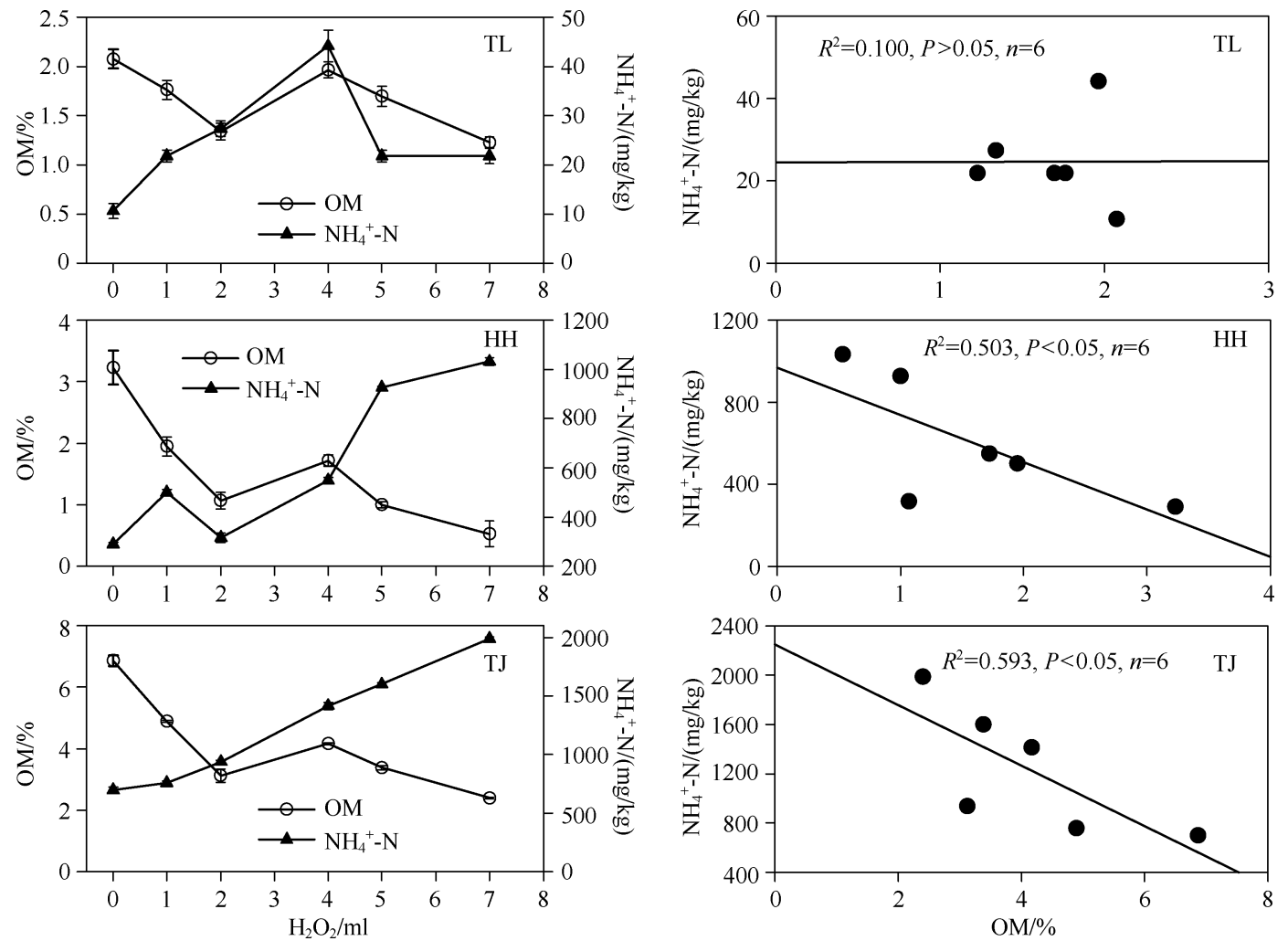

图 2 处理后沉积物有机质和铵态氮含量以及二者的相关关系

Fig. 2 Contents of $\mathrm{OM}, \mathrm{NH}_{4}^{+}-\mathrm{N}$ and the correlation relationship between $\mathrm{NH}_{4}^{+}-\mathrm{N}$ and $\mathrm{OM}$ in the treatment sediments

后减缓至平衡的趋势, 快速释放后各组均出现不同程度的吸附现象 (图 3). $\mathrm{HH}$ 组和 TJ 组大致在 $30 \mathrm{~min}$ 内 达到释放平衡, TL 组大致在 $60 \mathrm{~min}$ 内释放达到平衡. 达到释放平衡所需时间要少于海洋沉积物 ${ }^{[19]}$ 和湖泊沉 积物铵态氮释放平衡时间 ${ }^{[16]}$. 运用一级动力学方程对铵态氮动力学释放过程进行模拟, 结果显示拟合精度 较高 $\left(r\right.$ 为 $0.848 \sim 0.989$ ), 通过方程拟合的各组 $N_{0}$ 值 (释放平衡时铵态氮的释放量) 见图 4a. 各组 $N_{0}$ 值随 $\mathrm{H}_{2} \mathrm{O}_{2}$ 使用量增加均呈上升趋势. $\mathrm{HH}$ 组和 TJ 组 $N_{0}$ 最大值出现在 $\mathrm{H}_{2} \mathrm{O}_{2}$ 使用量为 $7 \mathrm{ml}$ 时, TL 组出现在 $\mathrm{H}_{2} \mathrm{O}_{2}$
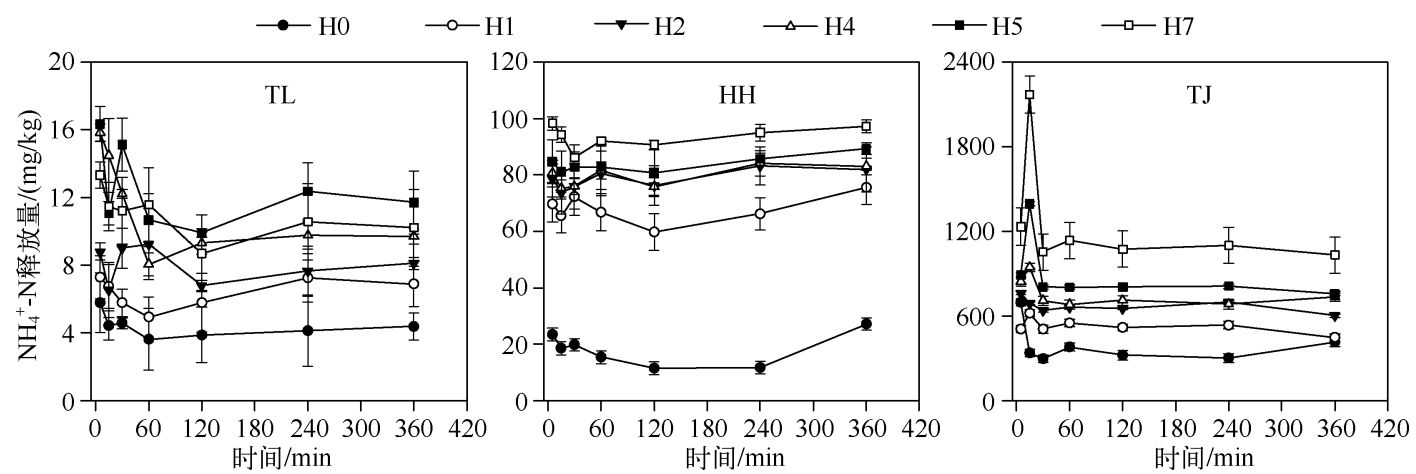

图 3 处理后沉积物铵态氮释放动力学曲线 ( $\mathrm{H} 0$ 表示 $\mathrm{H}_{2} \mathrm{O}_{2}$ 使用量为 $0 \mathrm{ml}, \mathrm{H} 1 、 \mathrm{H} 2 \cdots$ 以此类推, 下同)

Fig. 3 Kinetic curves of $\mathrm{NH}_{4}^{+}-\mathrm{N}$ release on different treatment sediments 
使用量为 $5 \mathrm{ml}$ 处, 各实验组 $N_{0}$ 最大值分别是处理前的 3.2、5.1 和 2.8 倍. 从数值上看, 沉积物本底有机质和 铵态氮含量最高的 TJ 组要明显高于 HH 组和 TL 组, 最大 $N_{0}$ 值分别是两组的 13.5 和 101.2 倍. HH 组和 TJ 组 $N_{0}$ 值与有机质存在显著负相关关系, 沉积物有机质本底值小的 TL 组相关性不显著 (图 4b). HH 组和 TJ 组 $N_{0}$ 值与铵态氮同样存在显著负相关关系, TL 组相关性不显著 (图 4c).

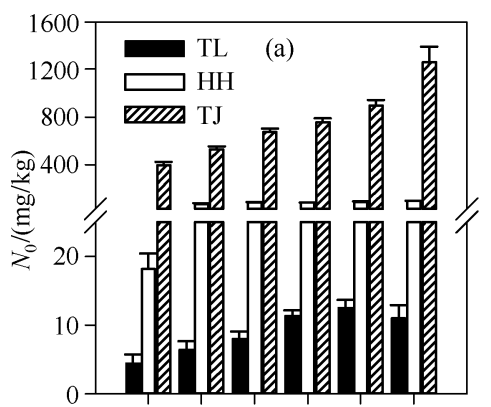

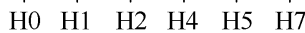
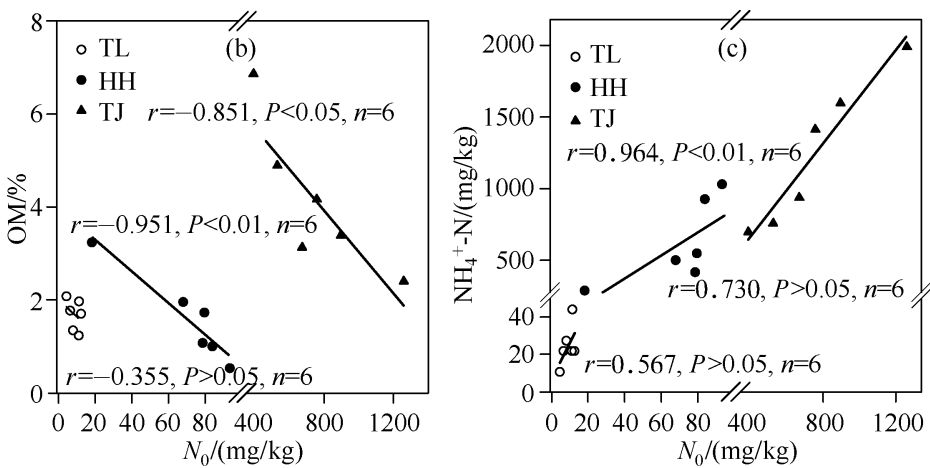

图 4 处理后沉积物铵态氮最大释放量 (a) 及与有机质 $(b)$ 、铵态氮 $(\mathrm{c})$ 的相关关系

Fig. 4 Variation value of $N_{0}($ a $)$, and its correlation relationship with $\mathrm{OM}$ (b) and $\mathrm{NH}_{4}^{+}-\mathrm{N}(\mathrm{c})$

in the treatment sediments

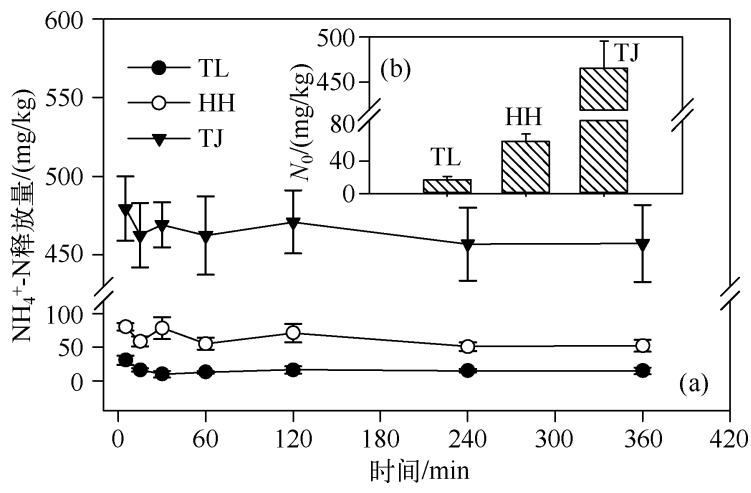

图 5 原沉积物铵态氮释放动力学曲线

Fig. 5 Kinetic curves of $\mathrm{NH}_{4}^{+}-\mathrm{N}$

release on original sediments
2.2.2 未经 $\mathrm{H}_{2} \mathrm{O}_{2}$ 处理沉积物 未经 $\mathrm{H}_{2} \mathrm{O}_{2}$ 处理 的原沉积物铵态氮释放动力学过程同样表现 出先快速释放, 后减缓至平衡趋势 (图 5 ). $\mathrm{HH}$ 组和 TJ 组大致在 $120 \mathrm{~min}$ 内达到释放平衡, TL 组大致在 $30 \mathrm{~min}$ 内释放达到平衡 (图 5a). 运 用一级动力学方程对铵态氮释放过程进行模 拟 $(P<0.05)$, 结果见图 5b. 拟合的各组 $N_{0}$ 值 表现为 $\mathrm{TJ}>\mathrm{HH}>\mathrm{TL}$, TJ 组分别是 $\mathrm{HH}$ 组和 TL 组的 7.2 倍和 64.3 倍. 铵态氮释放量呈现出 随着有机质含量增加而增加的趋势, 与 $\mathrm{H}_{2} \mathrm{O}_{2}$ 处 理组的变化趋势正好相反.

\section{3 有机质对沉积物铵态氮吸附热力学的} 影响

各实验组沉积物对铵态氮的吸附量总体 上随铵态氮标准液浓度增加而增大, 在低浓度 范围内, 沉积物对铵态氮的吸附量呈线性增长 (图 6a), 与相关研究相似 ${ }^{[20]}$. 在设定的浓度范围内, 沉积物对 铵态氮的吸附量仍然有上升的趋势. 经 $\mathrm{H}_{2} \mathrm{O}_{2}$ 处理后随着有机质减少, 各组沉积物对铵态氮的吸附能力明显 降低 $(P<0.01)$. 用 Langmuir 等温吸附方程拟合沉积物对铵态氮的饱和吸附量 $\left(Q_{\mathrm{m}}\right)$, 拟合精度符合要求 $(r$ 为 $0.788 \sim 0.957$ ), 结果见图 $6 \mathrm{~b}$. TJ 组 $Q_{\mathrm{m}}$ 值分别是 $\mathrm{HH}$ 组和 $\mathrm{TL}$ 组的 1.2 倍和 1.7 倍; 经 $\mathrm{H}_{2} \mathrm{O}_{2}$ 处理后 $\mathrm{TJH}$ 组 $Q_{\mathrm{m}}$ 值最大, $\mathrm{HHH}$ 组最小 ( HHH 组有机质含量最小).

\section{3 讨论}

\section{3. $1 \mathrm{H}_{2} \mathrm{O}_{2}$ 处理对沉积物有机质的影响}

沉积物中的有机质在氧化环境中可以被直接氧化分解, 而在还原性环境中则会在微生物作用下经过复 杂的生化过程形成较为稳定的腐殖质 ${ }^{[21]}$, 腐殖质一般占总有机质含量的 $50 \%$ 以上. 根据腐殖质氧化稳定 性, 一般将腐殖质分为活性腐殖质和非活性腐殖质 ${ }^{[22]}$, 活性腐殖质的分子结构较为简单、分子量较小、含有 

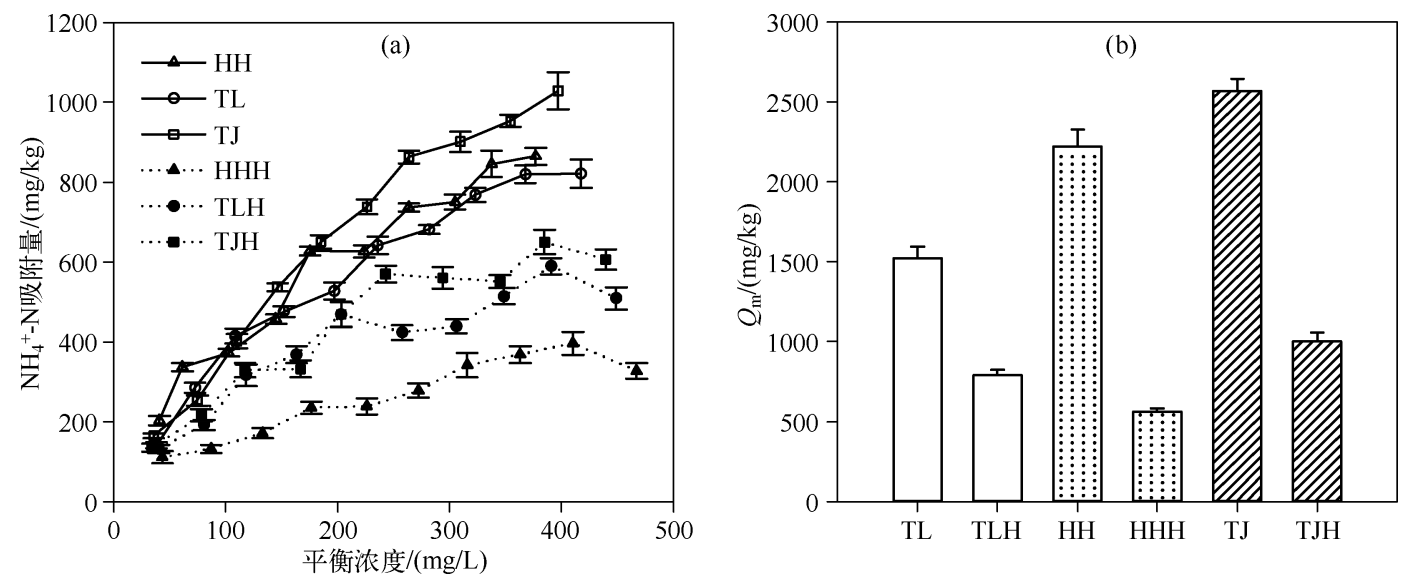

图 6 沉积物对 $\mathrm{NH}_{4}^{+}-\mathrm{N}$ 的等温吸附曲线 (a) 和饱和吸附量 $(b)$

Fig. 6 Adsorption isotherms curve(a) and adsorption capacity(b) of ammonium on the sediments

较多极性官能团, 容易被氧化分解 ${ }^{[23-24]}$. 在自然条件下, 有机质在腐殖化过程中一般先形成分子量较小、结 构较为简单的活性腐殖质, 随着腐殖化进程加深, 活性腐殖质趋向于形成结构更紧密和疏水性更强的高分 子聚合物, 即非活性腐殖质 ${ }^{[25-26]}$.

$\mathrm{H}_{2} \mathrm{O}_{2}$ 对有机质的氧化具有选择性,一般先氧化含氮、芳香族、烷烃链等极性官能团的有机质 ${ }^{[11]}$, 大多为 新富集的有机质以及活性腐殖质. 各实验组单位体积 $\mathrm{H}_{2} \mathrm{O}_{2}$ 对有机质去除效率随着 $\mathrm{H}_{2} \mathrm{O}_{2}$ 使用量的增加而降 低 (图 2), 表明沉积物有机质在逐渐被 $\mathrm{H}_{2} \mathrm{O}_{2}$ 氧化的过程中, 新富集于沉积物的有机质以及腐殖化进程较浅 的活性腐殖质首先被氧化; 随着有机质减少,有机质的组成结构发生变化,趋向于结构非常紧密的非活性腐 殖质, 导致有机质越难被氧化分解 ${ }^{[16]}$. 各实验组之间有机质在随 $\mathrm{H}_{2} \mathrm{O}_{2}$ 使用量被去除进程的差异, 表明各河 段沉积物有机质形成时间和腐殖化程度存在差异. TJ 组在 $\mathrm{H}_{2} \mathrm{O}_{2}$ 使用量为 $2 \mathrm{ml}$ 时的单位质量沉积物有机质 比沉积物本底有机质下降了 $37383.78 \mathrm{mg}$, 下降数值明显大于其他 2 组 (图 2). 可见, 采集于接纳生活污水的 河道 TJ 组沉积物, 有机质多为新富集于表面的来源于生活废水的简单有机质, 这类有机质一般为易被分解 的芳香性的生活油脂和清洁试剂 ${ }^{[16]}$. 由于 TJ 组的氧化还原条件较差 (表 1), 因此有机质的氧化分解较为缓 慢,造成了沉积物新鲜有机质短期内的大量累积,这可能是导致 TJ 组有机质去除量最大的主要原因. 濠河最 近一次清淤工程在 2009 年完成, HH 组沉积物多为新聚集的沉积物. 由于濠河是南通市的标志景观, 濠河周 边的污染源已得到有效控制, 其有机质来源较通甲河 ( TJ 组) 要少得多, 富集于沉积物的有机质量要少于 TJ 组, 而且濠河氧化条件较通甲河要优越 (表 1), 因此 HH 组有机质去除量要小于 TJ 组 (图 2). 通吕运河开丵 历史悠久, 与长江进行着频繁且较大的水量交换,水体氧化还原环境较好 (表 1), 沉积物一水界面在 DO 作用 下, 表层沉积物中新形成的有机质易被氧化分解而不宜富集. 那么, 在水力、DO 等因素的作用下集留在通吕 运河河道内的沉积物 ( TL 组) 有机质经过长时间的演化大多腐殖化程度高, 不易被氧化分解, 导致 $\mathrm{H}_{2} \mathrm{O}_{2}$ 对 TL 组有机质去除率最低 (图 2). 经 $\mathrm{H}_{2} \mathrm{O}_{2}$ 处理后的沉积物有机质组分和腐殖化程度均发生了变化, 对铵态氮 的吸附一解吸会产生影响.

表 1 采样点上覆水主要理化指标

Tab. 1 Physico-chemical characteristics of overlying water in study sites

\begin{tabular}{ccccccc}
\hline 采样点 & $\begin{array}{c}\mathrm{DO} / \\
(\mathrm{mg} / \mathrm{L})\end{array}$ & $\mathrm{pH}$ & $\mathrm{COD}_{\mathrm{Mn}} /(\mathrm{mg} / \mathrm{L})$ & $\begin{array}{c}\mathrm{TN} / \\
(\mathrm{mg} / \mathrm{L})\end{array}$ & $\begin{array}{c}\mathrm{NH}_{4}^{+}-\mathrm{N} / \\
(\mathrm{mg} / \mathrm{L})\end{array}$ & $\begin{array}{c}\mathrm{NO}_{3}^{-}-\mathrm{N}+\mathrm{NO}_{2}^{-}-\mathrm{N} / \\
(\mathrm{mg} / \mathrm{L})\end{array}$ \\
\hline $\mathrm{TL}$ & $4.98 \pm 0.23$ & $7.90 \pm 0.04$ & $3.48 \pm 0.41$ & $3.25 \pm 0.31$ & $0.85 \pm 0.09$ & $2.26 \pm 0.16$ \\
$\mathrm{HH}$ & $4.52 \pm 0.79$ & $7.96 \pm 0.23$ & $5.01 \pm 0.54$ & $4.84 \pm 0.24$ & $2.49 \pm 0.13$ & $1.34 \pm 0.14$ \\
$\mathrm{TJ}$ & $0.33 \pm 0.02$ & $7.81 \pm 0.03$ & $12.23 \pm 0.56$ & $14.26 \pm 0.11$ & $11.23 \pm 0.10$ & $1.84 \pm 0.11$ \\
\hline
\end{tabular}




\section{2 有机质对沉积物铵态氮吸附-解吸的影响}

在自然环境中沉积物铵态氮吸附一解吸过程是一个复杂的物理化学过程, 受到许多因素影响, 其中, 有 机质被认为是制约沉积物铵态氮吸附一解吸过程的关键因素 ${ }^{[5-6,27-30]}$. 有机质中存在着许多具有极性的官能 团, 这些官能团使沉积物颗粒带有大量的表面负电荷 ${ }^{[15]}$, 从而对铵态氮具有较强的吸附能力. 研究已发现 $\mathrm{H}_{2} \mathrm{O}_{2}$ 对有机质的去除具有一定的选择性, 一般先氧化含极性官能团的有机质, 主要是含氧、氮有机物和芳香 化合物 ${ }^{[11]}$. 随着有机质的去除极性官能才也随之减少 ${ }^{[16]}$, 沉积物对铵态氮的吸附能力减弱, 这可能是造成 各实验组沉积物对铵态氮的饱和吸附量随有机质含量减少而下降的主要原因 (图 6b).

经 $\mathrm{H}_{2} \mathrm{O}_{2}$ 处理沉积物铵态氮释放动力学实验中发现随着有机质减少, 沉积物对铵态氮吸附能力减弱, 铵 态氮的解吸释放量加大 (图 4). 但是, 未经 $\mathrm{H}_{2} \mathrm{O}_{2}$ 处理的原沉积物铵态氮释放动力学实验却表现出相反的结 果 (图 5). 这表明沉积物铵态氮的解吸释放过程除了受有机质含量的影响, 还受到沉积物铵态氮含量的影 响. 释放动力学的实验方法是通过一定时间内钾离子置换铵离子的量来计算铵态氮释放量, 那么, 沉积物吸 附态铵态氮从理论上讲都有可能成为潜在的铵态氮释放源, 且铵态氮含量越高潜在的铵态氮释放量亦可能 越大. 经 $\mathrm{H}_{2} \mathrm{O}_{2}$ 处理后, 各实验组沉积物铵态氮含量都有了不同程度的增加, 尤其是本底有机质含量较高的 $\mathrm{TJ}$ 组和 $\mathrm{HH}$ 组, 铵态氮含量与有机质含量存在显著的负相关关系 (图 2). 说明在 $\mathrm{H}_{2} \mathrm{O}_{2}$ 氧化有机质同时促进 了沉积物有机氮氨化进程, 表明 $\mathrm{H}_{2} \mathrm{O}_{2}$ 对含氮有机物氧化作用明显 ${ }^{[11]}$, 王圣瑞等 ${ }^{[16]}$ 也发现了同样的现象. 同 时还发现 TJ 组和 $\mathrm{HH}$ 组铵态氮含量与 $N_{0}$ 值呈显著相关 (图 4c) , 表明在短时间内经氨化作用形成的大量铵 态氮是促进沉积物铵态氮释放的一个不容忽视的原因. 未经 $\mathrm{H}_{2} \mathrm{O}_{2}$ 处理的原沉积物铵态氮含量随着有机质含 量的减少而下降, 表现为 $\mathrm{TJ}>\mathrm{HH}>\mathrm{TL}$ (表 2). 表明在自然环境下, 在漫长的矿化过程中随着有机质的减少, 通过有机氮矿化形成的铵态氮, 在一定条件下通过解吸作用进人水相或者转化为其他形态, 并没有大量富 集于沉积物中. 那么, 随着沉积物有机质含量减少对铵态氮吸附能力减弱, 本底吸附态铵态氮含量也在减 少, 潜在铵态氮的释放量也随之下降. 城市污染河道尤其是严重污染的黑臭河道, 沉积物中往往都富集了大 量的新生有机质. 在进行河道整治时, 应防止快速削减沉积物有机质而出现的有机氮氨化导致的铵态氮加 速释放的问题.

表 2 采样点沉积物和间隙水主要理化指标

Tab. 2 Physico-chemical characteristics of sediment and porewater in study sites

\begin{tabular}{|c|c|c|c|c|c|c|c|c|}
\hline \multirow[b]{2}{*}{ 采样点 } & \multirow[b]{2}{*}{ 颜色 } & \multirow[b]{2}{*}{$\mathrm{OM} / \%$} & \multicolumn{3}{|c|}{ 沉积物 / (mg/kg) } & \multicolumn{3}{|c|}{ 间隙水 $/(m g / L)$} \\
\hline & & & $\mathrm{TN}$ & $\mathrm{NH}_{4}^{+}-\mathrm{N}$ & $\mathrm{NO}_{3}^{-}-\mathrm{N}$ & $\mathrm{TN}$ & $\mathrm{NH}_{4}^{+}-\mathrm{N}$ & $\begin{array}{c}\mathrm{NO}_{3}^{-}-\mathrm{N}+ \\
\mathrm{NO}_{2}^{-}-\mathrm{N}\end{array}$ \\
\hline $\mathrm{TL}$ & 棕色 & $2.06 \pm 0.12$ & $1736.37 \pm 25.49$ & $10.61 \pm 3.78$ & $62.34 \pm 13.27$ & $9.52 \pm 0.62$ & $6.71 \pm 0.02$ & $0.86 \pm 0.13$ \\
\hline HH & 褐色 & $3.23 \pm 0.10$ & $2433.67 \pm 89.67$ & $290.43 \pm 21.23$ & $21.65 \pm 7.86$ & $15.63 \pm 1.07$ & $11.74 \pm 0.75$ & $0.67 \pm 0.11$ \\
\hline $\mathrm{TJ}$ & 黑色 & $6.87 \pm 0.23$ & $3830.23 \pm 280.44$ & $697.95 \pm 40.41$ & $12.56 \pm 3.26$ & $60.07 \pm 7.34$ & $52.36 \pm 5.17$ & $0.21 \pm 0.07$ \\
\hline
\end{tabular}

综上所述, $\mathrm{H}_{2} \mathrm{O}_{2}$ 对沉积物有机质具有一定的氧化性, 随着 $\mathrm{H}_{2} \mathrm{O}_{2}$ 使用量增加有机质有减少趋势; $\mathrm{H}_{2} \mathrm{O}_{2}$ 对 有机质的氧化具有选择性, 单位体积 $\mathrm{H}_{2} \mathrm{O}_{2}$ 对有机质去除效率随着 $\mathrm{H}_{2} \mathrm{O}_{2}$ 使用量的增加而降低; $\mathrm{H}_{2} \mathrm{O}_{2}$ 氧化有 机质的同时促进了沉积物有机氮氨化进程, $\mathrm{H}_{2} \mathrm{O}_{2}$ 对含氮有机物氧化作用明显. 经 $\mathrm{H}_{2} \mathrm{O}_{2}$ 处理的沉积物对辖态 氮的饱和吸附量随有机质含量减少而下降, 沉积物对轱态氮的最大释放量随有机质含量减少而呈增大趋 势; 沉积物铵态氮释放过程均表现为先快速释放, 后减缓至平衡过程. 沉积物有机质结构及组成和铵态氮本 底含量是影响沉积物铵态氮释放的主要因素. 针对城市污染河道的沉积物进行治理时, 短期内削减沉积物 有机质可能会出现由于有机氮氨化而导致的铵态氮加速释放的问题.

\section{4 参考文献}

[ 1 ] Mackin JE, Aller RC. Ammonium adsorption in marine sediments. Limnology and Oceanography, 1984,29(2):250-257.

[ 2 ] Eggleton J, Thomas KV. A review of factors affecting the release and bioavailability of contaminants during sediment disturbance events. Environment International, $2004,30(7)$ : 973-980. 
[ 3 ] 邢雅囡,阮晓红, 赵振华. 城市重污染河道环境因子对底质氮释放影响. 水科学进展,2010,21(1):120-126.

[ 4 ] 姜 霞,王秋娟, 王书航等. 太湖沉积物氮磷吸附一解吸特征分析. 环境科学,2011,32(5):1285-1291.

[ 5 ] Hou LJ, Liu M, Jiang HY et al. Ammonium adsorption by tidal flat surface sediments from the Yangtze Estuary. Environmental Geology, 2003,45(1):72-78.

[ 6 ] Boatman $\mathrm{CD}$, Murray JW. Modeling exchangeable $\mathrm{NH}_{4}^{+}$adsorption in marine sediments: process and controls of adsorption. Limnology and Oceanography, 1982,27 (1) :99-110.

[ 7 ] 刘 敏,侯立军, 许世远等. 长江口潮滩表层沉积物对 $\mathrm{NH}_{4}^{+}-\mathrm{N}$ 的吸附特征. 海洋学报: 中文版,2005,27(5):60-66.

[ 8 ] Holmboe N, Kristensen E. Ammonium adsorption in sediments of a tropical mangrove forest (Thailand) and a temperate Wadden Sea area (Denmark). Wetlands Ecology and Management, 2002,10 (6) : 453-460.

[ 9 ] Alongi DM. The dynamics of benthic nutrient pools and fluxes in tropical mangrove forests. Journal of Marine Research, $1996, \mathbf{5 4}(1): 123-148$.

[10] Nam K, Alexander M. Role of nanoporosity and hydrophobicity in sequestration and bioavailability: tests with model solids. Environmental Science and Technology, 1998,32(1):71-74.

[11] 梁重山,党 志, 刘丛强等. 土壤有机质对菲的吸附一解吸平衡的影响. 高等学校化学学报,2005,26(4):671-676.

[12］焦文涛, 蒋 新,余贵芬等. 土壤有机质对镉在土壤中吸附一解吸行为的影响. 环境化学,2005,24(5):545-549.

[13] 李祥平, 张飞, 齐剑英等. 土壤有机质对铊在土壤中吸附一解吸行为的影响. 环境工程学报, 2012,6 (11): 4245-4250.

[14] 朱 宁, 颜 丽, 张晓静等. 有机质对 $\mathrm{Cu}^{2+}$ 在棕壤及其各粒级微团聚体中吸附解吸特性的影响. 生态环境学报, $2009, \mathbf{1 8}(2): 498-501$.

[15] 鲍艳宇, 周启星, 莹 万等. 土壤有机质对土霉素在土壤中吸附一解吸的影响. 中国环境科学, 2009,29 (6): 651-655.

[16] 王圣瑞, 赵海超, 王 娟等. 有机质对湖泊沉积物不同形态氮释放动力学影响研究. 环境科学学报, 2012,32(2): 332-340.

[17] 古小治,王 强,张 雷等. 物理改良对湖泊沉积物和间隙水特征的影响. 中国环境科学,2010,30(2):256-262.

[18] Wang J, Wang S, Jin X et al. Ammonium release characteristics of the sediments from the shallow lakes in the middle and lower reaches of Yangtze River region, China. Environmental Geology, 2007,55(1):37-45.

[19] Morin J, Morse JW. Ammonium release from resuspended sediments in the Laguna Madre estuary. Marine Chemistry, $1999, \mathbf{6 5}(1 / 2): 97-110$.

[20] 王 娟,王圣瑞,金相灿等. 长江中下游浅水湖泊表层沉积物对氨氮的吸附特征. 农业环境科学学报,2007,26(4): 1224-1229.

[21] 易文利,王圣瑞, 杨苏文等. 长江中下游浅水湖泊沉积物腐殖质组分赋存特征. 湖泊科学, 2011,23(1):21-28.

[22] 王 晶,解宏图,朱 平等. 土壤活性有机质(碳) 的内涵和现代分析方法概述. 生态学杂志,2003,22(6):109-112.

[23] 鲁如坤,朱海舟,何平安等.土壤农业化学分析方法. 北京:中国农业科技出版社,2000.

[24] Blair GJ, Lefroy RD, Lisle L. Soil carbon fractions based on their degree of oxidation, and the development of a carbon management index for agricultural systems. Crop and Pasture Science, 1995,46(7) :1459-1466.

[25] Krosshavn M, Southon TE, Steinnes E. The influence of vegetational origin and degree of humification of organic soils on their chemical composition, determined by solid-state ${ }^{13}$ C NMR. Journal of Soil Science, $1992, \mathbf{4 3}(3)$ : 485-493.

[26] Tardy Y, Schaul R, Duplay J. Thermodynamic stability fields of humus, microflora and plants. Comptes Rendus de l' Académie des Sciences-Series IIA-Earth and Planetary Science, 1997,324(12):969-976.

[27] Rosenfeld JK. Ammonium adsorption in nearshore anoxic sediments. Limnology and Oceanography, 1979, 24 (2): 356-364.

[28 ] Wang J, Zhang Y. The effects of organic matter on ammonium adsorption in Wuli Lake sediments. In: Computer Distributed Control and Intelligent Environmental Monitoring (CDCIEM). Changsha,2011.

[29] 侯立军,刘 敏,蒋海燕等. 河口潮滩沉积物对氨氮的等温吸附特性. 环境化学, 2003,22(6):568-572.

[30] 王而力, 王嗣淇, 薛 扬. 沉积物不同天然有机组分对氨氮吸附特征的影响. 生态与农村环境学报,2012,28(5): 544-549. 